\title{
On a new poverty measure constructed from the exponential mean
}

\author{
S. Bortot ${ }^{1}$ R. A. Marques Pereira ${ }^{2}$ \\ ${ }^{1}$ Department of Economics and Management, University of Trento, Italy \\ ${ }^{2}$ Department of Economics and Management, University of Trento, Italy
}

\begin{abstract}
We propose a poverty measure based on a non trivial balance between the aggregated value of the income gaps of the poor and the headcount ratio of the poor in the population. The new poverty measure extends a previous proposal also based on the exponential mean but with an exclusive focus on the poor sector of the income distribution.
\end{abstract}

Keywords: Averaging functions, welfare and inequality, exponential means, poverty measures, aggregation functions, focus and interaction.

\section{Welfare functions and inequality indices}

We consider populations of $n \geq 2$ individuals and we briefly review the notions of welfare function and inequality index in the standard framework of averaging functions on the $\mathbb{D}^{n}$ domain, with $\mathbb{D}=[0, \infty)$. Comprehensive reviews of averaging functions can be found in Fodor and Roubens [22], Calvo et al. [15], Beliakov et al. [5], and Grabisch et al. [25].

The income distributions in this framework are represented by points $\boldsymbol{x}, \boldsymbol{y} \in \mathbb{D}^{n}$. In any case, most of our results hold analogously over different domains, for instance the reduced domain $[0,1]$ or even the extended domain $\mathbb{R}$.

Notation. Points in $\mathbb{D}^{n}$ are denoted $\boldsymbol{x}=$ $\left(x_{1}, \ldots, x_{n}\right)$, with $\mathbf{1}=(1, \ldots, 1), \mathbf{0}=(0, \ldots, 0)$. Accordingly, for every $x \in \mathbb{D}$, we have $x \cdot \mathbf{1}=$ $(x, \ldots, x)$. Given $\boldsymbol{x}, \boldsymbol{y} \in \mathbb{D}^{n}$, by $\boldsymbol{x} \geq \boldsymbol{y}$ we mean $x_{i} \geq y_{i}$ for every $i=1, \ldots, n$, and by $\boldsymbol{x}>\boldsymbol{y}$ we mean $\boldsymbol{x} \geq \boldsymbol{y}$ and $\boldsymbol{x} \neq \boldsymbol{y}$. Given $\boldsymbol{x} \in \mathbb{D}^{n}$, the increasing and decreasing reorderings of the coordinates of $\boldsymbol{x}$ are indicated as $x_{(1)} \leq$ $\cdots \leq x_{(n)}$ and $x_{[1]} \geq \cdots \geq x_{[n]}$, respectively. In particular, $x_{(1)}=\min \left\{x_{1}, \ldots, x_{n}\right\}=x_{[n]}$ and $x_{(n)}=\max \left\{x_{1}, \ldots, x_{n}\right\}=x_{[1]}$. In general, given a permutation $\sigma$ on $\{1, \ldots, n\}$, we denote $\boldsymbol{x}_{\sigma}=$ $\left(x_{\sigma(1)}, \ldots, x_{\sigma(n)}\right)$. Finally, the arithmetic mean is denoted $\bar{x}=\left(x_{1}+\cdots+x_{n}\right) / n$.

Definition 1 Let $A: \mathbb{D}^{n} \longrightarrow \mathbb{D}$ be a function.

1. $A$ is monotonic if $\boldsymbol{x} \geq \boldsymbol{y} \Rightarrow A(\boldsymbol{x}) \geq A(\boldsymbol{y})$, for all $\boldsymbol{x}, \boldsymbol{y} \in \mathbb{D}^{n}$. Moreover, $A$ is strictly monotonic if $\boldsymbol{x}>\boldsymbol{y} \Rightarrow A(\boldsymbol{x})>A(\boldsymbol{y})$, for all $\boldsymbol{x}, \boldsymbol{y} \in \mathbb{D}^{n}$.
2. $A$ is idempotent if $A(x \cdot \mathbf{1})=\mathbf{x}$, for all $x \in \mathbb{D}$. On the other hand, $A$ is nilpotent if $A(x \cdot \mathbf{1})=$ $\mathbf{0}$, for all $x \in \mathbb{D}$.

3. $A$ is symmetric if $A\left(\boldsymbol{x}_{\sigma}\right)=A(\boldsymbol{x})$, for any permutation $\sigma$ on $\{1, \ldots, n\}$ and all $\boldsymbol{x} \in \mathbb{D}^{n}$.

4. $A$ is invariant for translations if $A(\boldsymbol{x}+t \cdot \mathbf{1})=$ $\mathbf{A}(\boldsymbol{x})$, for all $t \in \mathbb{D}$ and $\boldsymbol{x} \in \mathbb{D}^{n}$. On the other hand, $A$ is stable for translations if $A(\boldsymbol{x}+t \cdot \mathbf{1})=$ $\mathbf{A}(\boldsymbol{x})+\mathbf{t}$, for all $t \in \mathbb{D}$ and $\boldsymbol{x} \in \mathbb{D}^{n}$.

5. A is invariant for dilations if $A(t \cdot \boldsymbol{x})=A(\boldsymbol{x})$, for all $t \in \mathbb{D}$ and $\boldsymbol{x} \in \mathbb{D}^{n}$. On the other hand, $A$ is stable for dilations if $A(t \cdot \boldsymbol{x})=t A(\boldsymbol{x})$, for all $t \in \mathbb{D}$ and $\boldsymbol{x} \in \mathbb{D}^{n}$.

We introduce the majorization relation on $\mathbb{D}^{n}$ and we discuss the concept of income transfer following the approach in Marshall and Olkin [26], focusing on the classical results relating majorization, income transfers, and bistochastic transformations, see Marshall and Olkin [26, Ch. 4, Prop. A.1].

Definition 2 The majorization relation $\preceq$ on $\mathbb{D}^{n}$ is defined as follows: given $\boldsymbol{x}, \boldsymbol{y} \in \mathbb{D}^{n}$ with $\bar{x}=\bar{y}$, we say that

$$
\boldsymbol{x} \preceq \boldsymbol{y} \quad \text { if } \quad \sum_{i=1}^{k} x_{(i)} \geq \sum_{i=1}^{k} y_{(i)} \quad k=1, \ldots, n
$$

where the case $k=n$ is an equality due to $\bar{x}=\bar{y}$. As usual, we write $\boldsymbol{x} \prec \boldsymbol{y}$ if $\boldsymbol{x} \preceq \boldsymbol{y}$ and not $\boldsymbol{y} \preceq \boldsymbol{x}$, and we write $\boldsymbol{x} \sim \boldsymbol{y}$ if $\boldsymbol{x} \preceq \boldsymbol{y}$ and $\boldsymbol{y} \preceq \boldsymbol{x}$. We say that $\boldsymbol{y}$ majorizes $\boldsymbol{x}$ if $\boldsymbol{x} \prec \boldsymbol{y}$, and we say that $\boldsymbol{x}$ and $\boldsymbol{y}$ are indifferent if $\boldsymbol{x} \sim \boldsymbol{y}$.

Another traditional reading, which reverses that of majorization, refers to the concept of Lorenz dominance: we say that $\boldsymbol{x}$ is Lorenz superior to $\boldsymbol{y}$ if $\boldsymbol{x} \prec \boldsymbol{y}$, and we say that $\boldsymbol{x}$ is Lorenz indifferent to $\boldsymbol{y}$ if $\boldsymbol{x} \sim \boldsymbol{y}$.

Given an income distribution $\boldsymbol{x} \in \mathbb{D}^{n}$, with mean income $\bar{x}$, it holds that $\bar{x} \cdot \mathbf{1} \preceq \boldsymbol{x}$ since $k \bar{x} \geq$ $\sum_{i=1}^{k} x_{(i)}$ for $k=1, \ldots, n$. The majorization is strict, $\overline{\boldsymbol{x}} \cdot \mathbf{1} \prec \boldsymbol{x}$, when $\boldsymbol{x}$ is not a uniform income distribution. In such case, $\bar{x} \cdot \mathbf{1}$ is Lorenz superior to $\boldsymbol{x}$. Moreover, for any income distribution $\boldsymbol{x} \in \mathbb{D}^{n}$ with mean income $\bar{x}$ it holds that $\boldsymbol{x} \preceq(0, \ldots, 0, n \bar{x})$, which is strict for $\boldsymbol{x} \neq \mathbf{0}$.

The majorization relation is a partial preorder, in the sense that $\boldsymbol{x}, \boldsymbol{y} \in \mathbb{D}^{n}$ are comparable only when 
$\bar{x}=\bar{y}$, and $\boldsymbol{x} \sim \boldsymbol{y}$ if and only if $\boldsymbol{x}$ and $\boldsymbol{y}$ differ by a permutation. In general, $\boldsymbol{x} \preceq \boldsymbol{y}$ if and only if there exists a bistochastic matrix $\mathbf{C}$ (non-negative square matrix of order $n$ where each row and column sums to one) such that $\boldsymbol{x}=\mathbf{C} \boldsymbol{y}$. Moreover, $\boldsymbol{x} \prec \boldsymbol{y}$ if the bistochastic matrix $\mathbf{C}$ is not a permutation matrix.

A particular case of bistochastic transformation is the so-called transfer, also called $T$-transformation.

Definition 3 Given $\boldsymbol{x}, \boldsymbol{y} \in \mathbb{D}^{n}$ with $\bar{x}=\bar{y}$, we say that $\boldsymbol{y}$ is derived from $\boldsymbol{x}$ by means of a income transfer $\mathrm{T}$, with $T(\boldsymbol{x})=\boldsymbol{y}$ if, for some pair $i, j=1, \ldots, n$ with $x_{i} \leq x_{j}$, we have

$y_{i}=(1-\varepsilon) x_{i}+\varepsilon x_{j} \quad y_{j}=\varepsilon x_{i}+(1-\varepsilon) x_{j} \quad \varepsilon \in[0,1]$

and $y_{k}=x_{k}$ for $k \neq i, j$. These formulas express an income transfer, from a richer to a poorer individual, of an income amount $\varepsilon\left(x_{j}-x_{i}\right)$. The income transfer obtains $\boldsymbol{x}=\boldsymbol{y}$ if $\varepsilon=0$, and exchanges the relative positions of donor and recipient in the income distribution if $\varepsilon=1$, in which case $\boldsymbol{x} \sim \boldsymbol{y}$. In the intermediate cases $\varepsilon \in(0,1)$ the income transfer produces an income distribution $\boldsymbol{y}$ which is Lorenz superior to the original $\boldsymbol{x}$, that is $\boldsymbol{x} \succ \boldsymbol{y}$.

In general, for the majorization relation $\preceq$ and income distributions $\boldsymbol{x}, \boldsymbol{y} \in \mathbb{D}^{n}$ with $\bar{x}=\bar{y}$, it holds that $\boldsymbol{x} \succeq \boldsymbol{y}$ if and only if $\boldsymbol{y}$ can be derived from $\boldsymbol{x}$ by means of a finite sequence of income transfers. Moreover, $\boldsymbol{x} \succ \boldsymbol{y}$ if any of the income transfers is not a permutation.

Definition 4 Let $A: \mathbb{D}^{n} \longrightarrow \mathbb{D}$ be a function. In relation with the majorization relation $\preceq$, the notions of Schur-convexity (S-convexity) and Schurconcavity (S-concavity) of the function $A$ are defined as follows:

1. A is S-convex if $\boldsymbol{x} \preceq \boldsymbol{y} \Rightarrow A(\boldsymbol{x}) \leq A(\boldsymbol{y})$ for all $\boldsymbol{x}, \boldsymbol{y} \in \mathbb{D}^{n}$

2. $A$ is S-concave if $\boldsymbol{x} \preceq \boldsymbol{y} \Rightarrow A(\boldsymbol{x}) \geq A(\boldsymbol{y})$ for all $\boldsymbol{x}, \boldsymbol{y} \in \mathbb{D}^{n}$.

Moreover, the S-convexity (resp. S-concavity) of a function $A$ is said to be strict if $\boldsymbol{x} \prec \boldsymbol{y}$ implies $A(\boldsymbol{x})<A(\boldsymbol{y})$ (resp. $A(\boldsymbol{x})>A(\boldsymbol{y})$ ). Notice that $S$-convexity (S-concavity) implies symmetry, since $\boldsymbol{x} \sim \boldsymbol{x}_{\sigma} \Rightarrow A(\boldsymbol{x})=A\left(\boldsymbol{x}_{\sigma}\right)$.

Definition 5 A function $A: \mathbb{D}^{n} \longrightarrow \mathbb{D}$ is an $n$-ary averaging function if it is monotonic and idempotent. An averaging function is said to be strict if it is strictly monotonic. Note that monotonicity and idempotency implies that $\min (\boldsymbol{x}) \leq A(\boldsymbol{x}) \leq \max (\boldsymbol{x})$, for all $\boldsymbol{x} \in \mathbb{D}^{n}$.

For simplicity, the $n$-arity is omitted whenever it is clear from the context. Particular cases of averaging functions are weighted averaging (WA) functions, ordered weighted averaging (OWA) functions, and Choquet integrals, which contain the former as special cases.
Definition 6 Given a weighting vector $\boldsymbol{w}=$ $\left(w_{1}, \ldots, w_{n}\right) \in[0,1]^{n}$, with $\sum_{i=1}^{n} w_{i}=1$, the Weighted Averaging (WA) function associated with $\boldsymbol{w}$ is the averaging function $A: \mathbb{D}^{n} \longrightarrow \mathbb{D}$ defined as

$$
A(\boldsymbol{x})=\sum_{i=1}^{n} w_{i} x_{i}
$$

Definition 7 Given a weighting vector $\boldsymbol{w}=$ $\left(w_{1}, \ldots, w_{n}\right) \in[0,1]^{n}$, with $\sum_{i=1}^{n} w_{i}=1$, the Ordered Weighted Averaging (OWA) function associated with $\boldsymbol{w}$ is the averaging function $A: \mathbb{D}^{n} \longrightarrow \mathbb{D}$ defined as

$$
A(\boldsymbol{x})=\sum_{i=1}^{n} w_{i} x_{(i)} .
$$

The traditional form of OWA functions as introduced by Yager [40] is as follows, $A(\boldsymbol{x})=$ $\sum_{i=1}^{n} \tilde{w}_{i} x_{[i]}$ where $\tilde{w}_{i}=w_{n-i+1}$. In $[41,42]$ the theory and applications of OWA functions are discussed in detail.

The following are two classical results particulary relevant in our framework. The proofs, given here for convenience, are analogous. The first result, see in particular Skala [36], regards a form of dominance relation between OWA functions, see also Bortot and Marques Pereira [13].

Proposition 1 Consider two OWA functions $A, B: \mathbb{D}^{n} \longrightarrow \mathbb{D}$ associated with weighting vectors $\boldsymbol{u}=\left(u_{1}, \ldots, u_{n}\right) \in[0,1]^{n}$ and $\boldsymbol{v}=\left(v_{1}, \ldots, v_{n}\right) \in[0,1]^{n}$, respectively. It holds that $A(\boldsymbol{x}) \leq B(\boldsymbol{x})$ for all $\boldsymbol{x} \in \mathbb{D}^{n}$ if and only if

$$
\sum_{i=1}^{k} u_{i} \geq \sum_{i=1}^{k} v_{i} \quad \text { for } \quad k=1, \ldots, n
$$

where the case $k=n$ is an equality due to weight normalization.

The next result, which is referred (without direct proof) by Weymark [37] and Chakravarty [16, p. 28], regards the relation between the weighting structure and the S-convexity or S-concavity of the OWA function, see also Bortot and Marques Pereira [13].

Proposition 2 Consider an OWA function $A$ : $\mathbb{D}^{n} \longrightarrow \mathbb{D}$ associated with a weighting vector $\boldsymbol{w}=$ $\left(w_{1}, \ldots, w_{n}\right) \in[0,1]^{n}$. The OWA function $A$ is $S$-convex if and only if the weights are non decreasing, $w_{1} \leq \cdots \leq w_{n}$, and $A$ is strictly $S$ convex if and only if the weights are increasing, $w_{1}<\cdots<w_{n}$. Analogously, the OWA function $A$ is $S$-concave if and only if the weights are non increasing, $w_{1} \geq \cdots \geq w_{n}$, and $A$ is strictly $S$ concave if and only if the weights are decreasing, $w_{1}>\cdots>w_{n}$.

We will now review the basic concepts and definitions regarding welfare functions and inequality 
indices. Certain properties which are generally considered to be inherent to the concepts of welfare and inequality are now accepted as basic axioms for welfare and inequality measures, see for instance Kolm [29, 30]. The crucial axiom in this field is the Pigou-Dalton transfer principle, which states that welfare (inequality) measures should be nondecreasing (non-increasing) under income transfers. This axiom translates directly into the properties of S-concavity and S-convexity in the context of symmetric functions on $\mathbb{D}^{n}$. In fact, a function is $\mathrm{S}$ concave (S-convex) if and only if it is symmetric and non-decreasing (non-increasing) under income transfers, see for instance Marshall and Olkin [26].

Definition 8 An averaging function $A: \mathbb{D}^{n} \longrightarrow \mathbb{D}$ is a welfare function if it is continuous, idempotent, and $S$-concave. The welfare function is said to be strict if it is a strict averaging function which is strictly $S$-concave.

Due to monotonicity and idempotency, a welfare function is non decreasing over $\mathbb{D}^{n}$ but increasing along the diagonal $\boldsymbol{x}=x \cdot \mathbf{1} \in \mathbb{D}^{n}$, with $x \in \mathbb{D}$. Moreover, notice that S-concavity implies symmetry. Due to S-concavity, a welfare function ranks any Lorenz superior income distribution with the same mean as $\boldsymbol{x}$ as no worse than $\boldsymbol{x}$, whereas a strict welfare function ranks it as better.

Given a welfare function $A$, the uniform equivalent income $\tilde{x}$ associated with an income distribution $\boldsymbol{x}$ is defined as the income level which, if equally distributed among the population, would generate the same welfare value, $A(\tilde{x} \cdot \mathbf{1})=A(\boldsymbol{x})$. The uniform equivalent concept has been originally proposed by Chisini [17] in the general context of averaging functions, see for instance Bennet et al. [6]. In the welfare context the uniform equivalent income has been considered by Atkinson [4], Kolm [28], and Sen [33] and further elaborated by Blackorby and Donaldson [8, 9, 10] and Blackorby, Donaldson, and Auersperg [12].

Due to the idempotency of $A$, we obtain $\tilde{x}=$ $A(\boldsymbol{x})$. Since $\bar{x} \cdot \mathbf{1} \preceq \boldsymbol{x}$ for any income distribution $\boldsymbol{x} \in \mathbb{D}^{n}$, S-concavity implies $A(\bar{x} \cdot \mathbf{1}) \geq A(\boldsymbol{x})$ and therefore $A(\boldsymbol{x}) \leq \bar{x}$ due to the idempotency of the welfare function. In other words, the mean income $\bar{x}$ and the uniform equivalent income $\tilde{x}$ are related by $0 \leq \tilde{x} \leq \bar{x}$.

We now define the notion of absolute inequality index, introduced by Kolm [29, 30] and developed by Blackorby and Donaldson [9], Blackorby, Donaldson, and Auersperg [12], and Weymark [37]. Following Kolm, inequality measures are described as "absolute" when they are invariant for additive transformations (translation invariance).

Definition 9 A function $G: \mathbb{D}^{n} \longrightarrow \mathbb{D}$ is an absolute inequality index if it is continuous, nilpotent, $S$-convex, and invariant for translations. The absolute inequality index is said to be strict if it is strictly $S$-convex.
In relation with the properties of the majorization relation discussed earlier, it holds that: over all income distributions $\boldsymbol{x} \in \mathbb{D}^{n}$ with the same mean income $\bar{x}$, a welfare function has minimum value $A(0, \ldots, 0, n \bar{x})$, and an absolute inequality index has maximum value $G(0, \ldots, 0, n \bar{x})$.

In the AKS framework introduced by Atkinson [4], Kolm [28], and Sen [33], a welfare function which is stable for translations induces an associated absolute inequality index by means of the correspondence formula $A(\boldsymbol{x})=\bar{x}-G(\boldsymbol{x})$, see Blackorby and Donaldson [9]. The welfare function and the associated inequality index are said to be ethical, see also Sen [35], Blackorby, Donaldson, and Auersperg [12], Weymark [37], Blackorby and Donaldson [11], and Ebert [20].

Definition 10 Given a welfare function $A$ : $\mathbb{D}^{n} \longrightarrow \mathbb{D}$ which is stable for translations, the associated Atkinson-Kolm-Sen (AKS) absolute inequality index $G: \mathbb{D}^{n} \longrightarrow \mathbb{D}$ is defined as

$$
G(x)=\bar{x}-A(x) .
$$

The fact that $A$ is stable for translations ensures the translational invariance of $G$. The absolute inequality index can be written as $G(\boldsymbol{x})=\bar{x}-\tilde{x}$ and represents the per capita income that could be saved if society distributed incomes equally without any loss of welfare.

In the AKS framework, a welfare function $A$ which is stable for both translations and dilations is associated with both absolute and relative inequality indices $G$ and $G_{R}$, respectively, with $G(\boldsymbol{x})=$ $\bar{x} G_{R}(\boldsymbol{x})$ for all $\boldsymbol{x} \in \mathbb{D}^{n}$. In what follows we will omit the term "absolute" when referring to $G$.

An important class of welfare functions which are stable for translations is that of the generalized Gini welfare functions introduced by Weymark [37], see also Mehran [31], Donaldson and Weymark [18, 19], Yaari [38, 39], Ebert [21], Quiggin [32], Ben-Porath and Gilboa [7].

Definition 11 Given a weighting vector $\boldsymbol{w}=$ $\left(w_{1}, \ldots, w_{n}\right) \in[0,1]^{n}$, with $w_{1} \geq \cdots \geq w_{n} \geq 0$ and $\sum_{i=1}^{n} w_{i}=1$, the generalized Gini welfare function associated with $\boldsymbol{w}$ is the function $A: \mathbb{D}^{n} \longrightarrow \mathbb{D}$ defined as

$$
A(\boldsymbol{x})=\sum_{i=1}^{n} w_{i} x_{(i)}
$$

and, in the AKS framework, the associated generalized Gini inequality index is defined as

$$
G(\boldsymbol{x})=\bar{x}-A(\boldsymbol{x})=-\sum_{i=1}^{n}\left(w_{i}-\frac{1}{n}\right) x_{(i)} .
$$

The generalized Gini welfare functions, which are strict if and only if $w_{1}>\cdots>w_{n}>0$, are clearly stable for both translations and dilations. For this reason they have a natural role within the AKS 
framework and Blackorby and Donaldson's correspondence formula.

A fundamental instance of the AKS generalized Gini framework is the classical Gini welfare function $A^{c}(\boldsymbol{x})$ and the associated classical Gini inequality index $G^{c}(\boldsymbol{x})=\bar{x}-A^{c}(\boldsymbol{x})$,

$$
A^{c}(\boldsymbol{x})=\sum_{i=1}^{n} \frac{2(n-i)+1}{n^{2}} x_{(i)}
$$

where the coefficients of $A^{c}(\boldsymbol{x})$ have unit sum, $\sum_{i=1}^{n}(2(n-i)+1)=n^{2}$, and

$$
G^{c}(\boldsymbol{x})=-\sum_{i=1}^{n} \frac{n-2 i+1}{n^{2}} x_{(i)}
$$

where the coefficients of $G^{c}(\boldsymbol{x})$ have zero sum, $\sum_{i=1}^{n}(n-2 i+1)=0$. The classical Gini inequality index $G^{c}$ is traditionally defined as

$$
G^{c}(\boldsymbol{x})=\frac{1}{2 n^{2}} \sum_{i, j=1}^{n}\left|x_{i}-x_{j}\right|,
$$

see for instance Bortot and Marques Pereira [13]. In this paper the authors discuss the family of binomial Gini welfare functions $C_{j}, j=1, \ldots, n$ and associated binomial Gini inequality indices $G_{j}$, $j=1, \ldots, n$. In particular, it is shown that $C_{2}$ and $G_{2}$ are proportional to the classical $A^{c}$ and $G^{c}$, respectively.

Another instance of the AKS correspondence between generalized Gini welfare functions and inequality indices is the S-Gini family introduced by Donaldson and Weymark [18], and independently by Kakwani [27] as an extension of a poverty measure proposed by Sen [34], see also Donaldson e Weymark [19], Yitzhaki [43], Bossert [14], and Aaberge [1, 2, 3].

\section{A new poverty measure}

In this section we introduce a new poverty measure based on a non trivial balance between the aggregated value of the income gaps of the poor and the headcount ratio of the poor in the population. This poverty measure extends a previous proposal also based on the exponential mean but with an exclusive focus on the poor sector of the income distribution.

The exponential mean is a strict averaging function which is symmetric and stable for translations. It is also decomposable, in the sense that the values associated with any given subset of individuals can each be substituted by their own aggregated value.

Definition 12 The exponential mean $F_{\alpha}: \mathbb{D}^{n} \rightarrow$ $\mathbb{D}$, with parameter $\alpha \in \mathbb{R}$, is defined as

$$
F_{\alpha}(\boldsymbol{x})=\frac{1}{\alpha} \ln \left(\frac{e^{\alpha x_{1}}+\cdots+e^{\alpha x_{n}}}{n}\right)
$$

for $\alpha \neq 0$, and $F_{\alpha=0}(\boldsymbol{x})=\bar{x}$.
The continuity of the exponential mean with respect to the parameter $\alpha$ is ensured by

$$
\lim _{\alpha \rightarrow 0} F_{\alpha}(\mathbf{x})=\frac{x_{1}+\cdots+x_{n}}{n}=\bar{x} .
$$

The following is a classical result, see for instance García-Lapresta et al. [24].

Proposition 3 The exponential mean $F_{\alpha}$ is $S$ convex (S-concave) for $\alpha \geq 0(\alpha \leq 0)$ and strictly $S$-convex (strictly $S$-concave) for $\alpha>0(\alpha<0)$.

Given an income distribution $\boldsymbol{x} \in \mathbb{D}^{n}$ and a poverty threshold $z \in(0, \infty)$ representing the necessary income to maintain a minimum level of living, the set of poor individuals in the population is identified by

$$
Q(\boldsymbol{x})=\left\{i \in\{1, \ldots, n\} \mid x_{i}<z\right\}
$$

and $q(\boldsymbol{x})=\# Q(\boldsymbol{x})$ is the number of the poor. We define the restricted poor income distribution $\boldsymbol{x}_{p}$ as

$$
x_{i}^{p}=x_{(i)} \quad i=1, \ldots, q
$$

where $q=q(\boldsymbol{x})$. In this way $x_{1}^{p} \leq x_{2}^{p} \leq \cdots \leq x_{q}^{p}$.

Given an income distribution $\boldsymbol{x} \in \mathbb{D}^{n}$ and a poverty threshold $z \in(0, \infty)$, the associated income gap distribution $g(\boldsymbol{x})=\left(g\left(x_{1}\right), \ldots, g\left(x_{n}\right)\right)$ is defined by means of the income gap function

$$
g(x)=\max \left(\frac{z-x}{z}, 0\right) \quad x \in \mathbb{D} .
$$

The income gap distribution is normalized in the sense that $g(x) \in[0,1]$ for any income $x \in \mathbb{D}$ and the income gaps of the non poor are null. Focusing on the poor we obtain the restricted poor income gap distribution $g\left(\boldsymbol{x}_{p}\right)$ as

$$
g\left(x_{i}^{p}\right)=g\left(x_{(i)}\right) \quad i=1, \ldots, q
$$

with $g\left(x_{1}^{p}\right) \geq g\left(x_{2}^{p}\right) \geq \cdots \geq g\left(x_{q}^{p}\right)$.

A poverty measure $P: \mathbb{D}^{n} \rightarrow[0,1]$ should satisfy the following traditional axioms:

- Poverty Focus (PF): For all $\boldsymbol{x}, \boldsymbol{y} \in \mathbb{D}^{n}$ and $z \in$ $(0, \infty)$, if $Q(\boldsymbol{x})=Q(\boldsymbol{y})=Q$ and $x_{i}=y_{i}$ for every $i \in Q$, then $P(\boldsymbol{x})=P(\boldsymbol{y})$.

- Poverty Monotonicity (PM): For all $\boldsymbol{x}, \boldsymbol{y} \in \mathbb{D}^{n}$ and $z \in(0, \infty)$, if $Q(\boldsymbol{x})=Q(\boldsymbol{y})=Q$ and $\boldsymbol{x}=\boldsymbol{y}$ except for $x_{i}>y_{i}$ with $i \in Q$, then $P(\boldsymbol{x})<$ $P(\boldsymbol{y})$.

- Transfer Sensitivity (TS): For all $\boldsymbol{x}, \boldsymbol{y} \in \mathbb{D}^{n}$ and $z \in(0, \infty)$, if $\boldsymbol{y}$ is obtained from $\boldsymbol{x}$ by an income transfer among the poor, with $\boldsymbol{x} \succ \boldsymbol{y}$, then $P(\boldsymbol{x})>P(\boldsymbol{y})$.

- Normalization $(\mathbf{N})$ : For all $\boldsymbol{x}, \boldsymbol{y} \in \mathbb{D}^{n}$ and $z \in$ $(0, \infty), P(\boldsymbol{x})=0$ if and only if $Q(\boldsymbol{x})=\emptyset$, that is $x_{i} \geq z$ for every $i \in\{1, \ldots, n\}$.

- Poverty Symmetry (PS): For all $\boldsymbol{x} \in \mathbb{D}^{n}, z \in$ $(0, \infty)$, and permutations $\sigma$ on $\{1, \ldots, n\}$, it holds that $P\left(\boldsymbol{x}_{\sigma}\right)=P(\boldsymbol{x})$. 
- Replication Invariance (RI): For all $\boldsymbol{x} \in \mathbb{D}^{n}$ and $z \in(0, \infty)$, if $\boldsymbol{y}$ is obtained from $\boldsymbol{x}$ by a replication, that is $\boldsymbol{y}=(\boldsymbol{x}, \ldots, \boldsymbol{x})$ with $m$ copies of the income distribution $\boldsymbol{x}$ for some $m \in \mathbb{N}$, then $P(\boldsymbol{y})=P(\boldsymbol{x})$.

- Diminishing Transfer Sensitivity (DTS): For all $\boldsymbol{x}, \boldsymbol{y} \in \mathbb{D}^{n}$ and $z \in(0, \infty)$, if $Q(\boldsymbol{x})=Q(\boldsymbol{y})$ and $\boldsymbol{y}$ is obtained from $\boldsymbol{x}$ by an income transfer from the poor person with income $x_{i}+c$ to the poor person with income $x_{i}$, for some $c>$ 0 , then the magnitude of decrease in poverty $P(\boldsymbol{x})-P(\boldsymbol{y})$ is higher the lower $x_{i}$.

On the basis of the exponential mean (12) and the income gap function (16) we introduce a new poverty measure depending on the full income gap distribution of the population. A preliminary version of this proposal has been presented in GarcíaLapresta et al. [23].

Definition 13 We define the poverty measure $P_{\alpha}$ : $\mathbb{D}^{n} \rightarrow[0,1]$, with parameter $\alpha \geq 0$, as

$$
P_{\alpha}(\boldsymbol{x})=F_{\alpha}(g(\boldsymbol{x}))
$$

which means

$$
P_{\alpha}(\boldsymbol{x})=\frac{1}{\alpha} \ln \left(\frac{e^{\alpha g\left(x_{1}\right)}+\cdots+e^{\alpha g\left(x_{n}\right)}}{n}\right)
$$

for $\alpha \neq 0$, and $P_{\alpha=0}(\boldsymbol{x})=\left(g\left(x_{1}\right)+\cdots+g\left(x_{n}\right)\right) / n$.

Proposition 4 For every $\alpha \geq 0$, the poverty measure $P_{\alpha}$ satisfies PF, PM, N, PS, RI. Moreover, $P_{\alpha}$ satisfies $\boldsymbol{T} \boldsymbol{S}$ and $\boldsymbol{D T} \boldsymbol{S}$ for every $\alpha>0$.

The poverty measure $P_{\alpha}$, which combines the income gap function and the exponential mean, is interesting in so far as it is analytically sensitive to the value of the poverty threshold as well as to income transfers between the rich and the poor, a form of extended transfer sensitivity, see also [23].

We can write the poverty measure as

$$
P_{\alpha}(\boldsymbol{x})=\frac{1}{\alpha} \ln \left(\frac{e^{\alpha g\left(x_{(1)}\right)}+\cdots+e^{\alpha g\left(x_{(q)}\right)}+n-q}{n}\right)
$$

since the $n-q$ income gaps of the non poor are null. We can now use the fact that the exponential mean is a decomposable aggregation function, see [22] [15] [5] [25], in order to obtain

$$
P_{\alpha}(\boldsymbol{x})=\frac{1}{\alpha} \ln \left(\frac{q e^{\alpha u_{p}}+n-q}{n}\right)
$$

where $u_{p}$ is the exponential mean of the income gaps of the poor, $u_{p}=F_{\alpha}\left(g\left(\boldsymbol{x}_{p}\right)\right)$, that is,

$$
u_{p}=\frac{1}{\alpha} \ln \left(\frac{e^{\alpha g\left(x_{(1)}\right)}+\cdots+e^{\alpha g\left(x_{(q)}\right)}}{q}\right)
$$

We can thus write the poverty measure $P_{\alpha}$ as

$$
P_{\alpha}(\boldsymbol{x})=f_{\alpha}(u, v)
$$

where $u=u_{p}$ is the aggregated value of the income gaps of the poor and $v=q / n$ is the headcount ratio of the poor in the income distribution $\boldsymbol{x}$. The aggregation function $f_{\alpha}$ is defined below.

Definition 14 We define the aggregation function $f_{\alpha}:[0,1]^{2} \rightarrow[0,1]$, with parameter $\alpha \geq 0$, as follows,

$$
f_{\alpha}(u, v)=\frac{1}{\alpha} \ln \left(1+\left(e^{\alpha u}-1\right) v\right)
$$

for $\alpha \neq 0$, and $f_{\alpha=0}(u, v)=u v$.

The continuity of the aggregation function with respect to the parameter $\alpha$ is ensured by

$$
\lim _{\alpha \rightarrow 0} f_{\alpha}(u, v)=u v .
$$

In the null parameter case the poverty measure reduces to $P_{\alpha=0}(\boldsymbol{x})=f_{\alpha=0}(u, v)=u v=u_{p}(q / n)$, which corresponds to the poverty measure proposed in García-Lapresta et al. [24]. The two poverty measures differ for positive values of the parameter $\alpha$, in which case the poverty measure $P_{\alpha}$ as in (23) breaks the $u, v$ symmetry which is present in [24] and yields a non trivial balance between the aggregated value of the income gaps of the poor and the headcount ratio of the poor in the population.

In the new poverty measure $P_{\alpha}$ as in (23) the behaviour of the aggregation function with respect to each variable is illustrated by the following figures:



Figure 1: $f_{\alpha}(u, v)$ as a function of $u$.

- Fig. 1 shows $f_{\alpha}(u, v)$ as a function of $u$ for two values of the variable $v: v=1 / 4$ (group below) and $v=3 / 4$ (group above). In each group the parameter $\alpha$ takes the values $\alpha=0,1,2,3,4$ where $\alpha=0$ corresponds to the inferior graph and $\alpha=4$ corresponds to the superior graph in the group.

- Fig. 2 shows $f_{\alpha}(u, v)$ as a function of $v$ for two values of the variable $u$ : $u=1 / 2$ (group below) and $u=1$ (group above). In each group the parameter $\alpha$ takes the values $\alpha=0,1,2,3,4$ where $\alpha=0$ corresponds to the inferior graph and $\alpha=4$ corresponds to the superior graph in the group. 


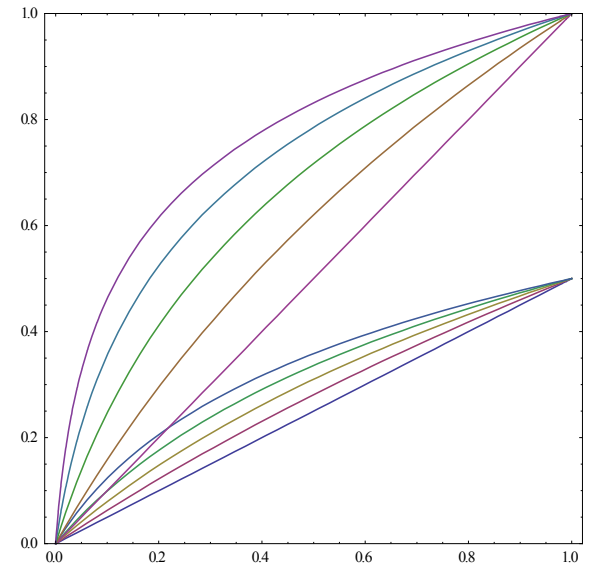

Figure 2: $f_{\alpha}(u, v)$ as a function of $v$.

From the interpretative point of view the parameter $\alpha$ tends to emphasize the convexity of the poverty measure dependence on $u$ and thus it tends to accentuate the importance of extreme poverty levels (high $u$ values). On the other hand the parameter $\alpha$ tends to emphasize the concavity of the poverty measure dependence on $v$ and thus it tends to accentuate the effect of scarce headcount ratios (low $v$ values).

The aggregation function $f_{\alpha}$ is continuously differentiable in any order with respect to the parameter. The derivatives of $f_{\alpha}$ with respect to the parameter at $\alpha=0$ can be obtained (by means of l'Hospital's rule) as follows,

$$
\begin{gathered}
\dot{f}_{\alpha=0}(u, v)=\lim _{\alpha \rightarrow 0} \frac{f_{\alpha}(u, v)-f_{\alpha=0}(u, v)}{\alpha-0} \\
\stackrel{\underline{H}}{=} \lim _{\alpha \rightarrow 0} \dot{f}_{\alpha}(u, v)=\frac{1}{2} u^{2} v(1-v) \\
\ddot{f}_{\alpha=0}(u, v)=\lim _{\alpha \rightarrow 0} \frac{\dot{f}_{\alpha}(u, v)-\dot{f}_{\alpha=0}(u, v)}{\alpha-0} \\
\stackrel{\underline{H}}{=} \lim _{\alpha \rightarrow 0} \ddot{f}_{\alpha}(u, v)=\frac{1}{3} u^{3} v(1-v)(1-2 v)
\end{gathered}
$$

just to mention the first and second order derivatives.

The general form of the derivatives of $f_{\alpha}$ with respect to the parameter at $\alpha=0$ is complex in its dependence on the variable $v$. However the roots $v=0$ and $v=1$ are always present in every order and the root $v=1 / 2$ is present in even orders. In Fig. 3 we indicate all the roots numerically obtained up to order sixteen. Notice the interesting sigmoid shape of the plot due to the fact that the root density is higher in the neighbourhood of the extreme values 0 and 1 .

Proposition 5 The aggregation function $f_{\alpha}$ as in (24), with parameter $\alpha \geq 0$, is strictly increasing in both variables $u$ and $v$, is strictly convex in $u$ and strictly concave in $v$, and overall it is neither concave nor convex.

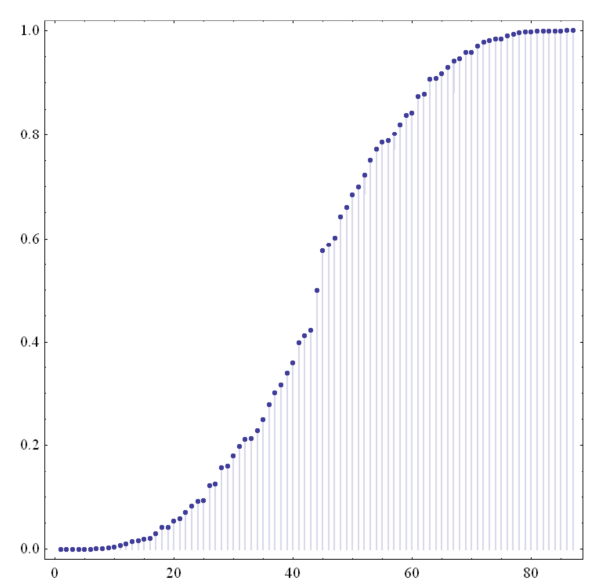

Figure 3: Roots in $v$ of derivatives of $f_{\alpha}$ w.r.t. $\alpha$.

Proof: The first partial derivatives of $f_{\alpha}$ with respect to the variables $u$ and $v$ are as follows,

$$
\begin{aligned}
\left(f_{\alpha}\right)_{u}^{\prime}(u, v) & =\frac{v}{v+(1-v) e^{-\alpha u}} \\
\left(f_{\alpha}\right)^{\prime}{ }_{v}(u, v) & =\frac{e^{\alpha u}-1}{\alpha\left(1+\left(e^{\alpha u}-1\right) v\right)}
\end{aligned}
$$

and continuity in the parameter $\alpha$ is ensured by

$$
\begin{aligned}
& \left(f_{\alpha=0}\right)_{u}^{\prime}(u, v)=v=\lim _{\alpha \rightarrow 0}\left(f_{\alpha}\right)_{u}^{\prime}(u, v) \\
& \left(f_{\alpha=0}\right)_{v}^{\prime}(u, v)=u=\lim _{\alpha \rightarrow 0}\left(f_{\alpha}\right)_{v}^{\prime}(u, v) .
\end{aligned}
$$

The second partial derivatives are as follows,

$$
\begin{gathered}
\left(f_{\alpha}\right){ }_{u u}^{\prime \prime}(u, v)=\frac{\alpha v(1-v)}{\left(v e^{\alpha u}+(1-v)\right)\left(v+(1-v) e^{-\alpha u}\right)} \\
\left(f_{\alpha}\right)^{\prime \prime}{ }_{v v}(u, v)=-\frac{\left(e^{\alpha u}-1\right)^{2}}{\alpha\left(1+\left(e^{\alpha u}-1\right) v\right)^{2}} \\
\left(f_{\alpha}\right)_{u v}^{\prime \prime}(u, v)=\frac{e^{\alpha u}}{\left(1+\left(e^{\alpha u}-1\right) v\right)^{2}}
\end{gathered}
$$

and continuity in the parameter $\alpha$ is ensured by

$$
\begin{gathered}
\left(f_{\alpha=0}\right)_{u u}^{\prime \prime}(u, v)=0=\lim _{\alpha \rightarrow 0}\left(f_{\alpha}\right){ }_{u u}^{\prime \prime}(u, v) \\
\left(f_{\alpha=0}\right)^{\prime \prime}{ }_{v v}(u, v)=0=\lim _{\alpha \rightarrow 0}\left(f_{\alpha}\right)^{\prime \prime}{ }_{v v}(u, v) \\
\left(f_{\alpha=0}\right)^{\prime \prime}{ }_{u v}(u, v)=1=\lim _{\alpha \rightarrow 0}\left(f_{\alpha}\right)^{\prime \prime}{ }_{u v}(u, v) .
\end{gathered}
$$

The determinant of the Hessian matrix of the aggregation function $f_{\alpha}$ is given by

$$
\operatorname{det} H_{\alpha}(u, v)=-\frac{v e^{-\alpha u}+(1-v)}{\left(v+(1-v) e^{-\alpha u}\right)^{3}} e^{-\alpha u}<0
$$

which means that the quadratic form associated with the Hessian matrix is indefinite, i.e., the aggregation function is neither concave nor convex. 
In relation with the balance between the variables $u$ and $v$ of the aggregation function $f_{\alpha}$, the points in which an increase in $u$ is compensated by an equal decrease in $v$ (or viceversa) are the solutions of the equation

$$
\begin{gathered}
\left(f_{\alpha}\right)_{u}^{\prime}(u, v)=\left(f_{\alpha}\right)_{v}^{\prime}(u, v) \\
\frac{v}{v+(1-v) e^{-\alpha u}}=\frac{1-e^{-\alpha u}}{\alpha\left(v+(1-v) e^{-\alpha u}\right)} \\
v=\frac{1}{\alpha}\left(1-e^{-\alpha u}\right)=h_{\alpha}(u)
\end{gathered}
$$

where the curve $v=h_{\alpha}(u)$, depending on the parameter $\alpha$, illustrates the character of the balancing mechanism between the two variables in the context of the new poverty measure. Notice that $h_{\alpha=0}(u)=u$ as in the poverty measure proposed in [24].

Finally, the Taylor expansion of the aggregation function $f_{\alpha}$ with respect to the parameter $\alpha$ provides further insight on the way the new poverty measure extends the one proposed in [24]. The Taylor expansion with respect to the parameter at $\alpha=0$ expresses the change in the aggregation mechanism of the poverty measure, particularly in the way it combines the two variables $u$ and $v$, from the symmetric balance $f_{\alpha=0}(u, v)=u v$ as in [24] to the more complex asymmetric balance in $f_{\alpha}(u, v)$ as in (24).

In this way the Taylor expansion with respect to the parameter illustrates the effect of having extended the focus of the poverty measure by considering the full income gap distribution of the population.

Below we indicate the second order Taylor expansion with respect to the parameter at $\alpha=0$,

$f_{\alpha}(u, v) \stackrel{\alpha}{\approx} u v\left(1+\frac{1}{2} \alpha u(1-v)\left(1+\frac{1}{3} \alpha u(1-2 v)\right)\right)$

where the null order term in the parameter corresponds to the case $f_{\alpha=0}(u, v)=u v$ as in the poverty measure [24]. The remaining terms,

- first order term $u^{2} v(1-v)$

- second order term $u^{3} v(1-v)(1-2 v)$

correspond to corrections which are proportional to increasing powers of $u$ (aggregated income gap of the poor) together with increasing order polynomials in $v$ whose roots introduce reference values for the headcount ratio of the poor in the population.

\section{Conclusion}

We propose a poverty measure based on the exponential mean of the full income gap distribution of the population. We show that this poverty measure expresses a non trivial balance between the aggregated value of the income gaps of the poor and the headcount ratio of the poor in the population.

The new poverty measure extends a previous proposal also based on the exponential mean but with an exclusive focus on the poor sector of the income distribution.

The new poverty measure combining the income gap function and the exponential mean is interesting in so far as it is analytically sensitive to the value of the poverty threshold as well as to income transfers between the rich and the poor, a form of extended transfer sensitivity.

\section{Acknowledgments}

The authors thank José Luis García-Lapresta and Casilda Lasso de la Vega for illuminating discussions and valuable comments.

\section{References}

[1] R. Aaberge, Characterization of Lorenz curves and income distributions, Social Choice and Welfare, 17:639-653, 2000.

[2] R. Aaberge, Axiomatic characterization of the Gini coefficient and Lorenz curve orderings, Journal of Economic Theory, 101:115-132, 2001.

[3] R. Aaberge, Gini's nuclear family, The Journal of Economic Inequality, 5:305-322, 2007.

[4] A. B. Atkinson, On the measurement of inequality, Journal of Economic Theory, 2:244263, 1970.

[5] G. Beliakov, A. Pradera and T. Calvo, Aggregation Functions: A Guide for Practitioners, Studies in Fuzziness and Soft Computing, Vol. 221, Springer, Heidelberg, 2007.

[6] C. D. Bennet, W. C. Holland and G. J. Székely, Integer valued means, Aequationes Mathematicae, 88:137-149, 2014.

[7] E. Ben Porath and I. Gilboa, Linear measures, the Gini index, and the income-equality tradeoff, Journal of Economic Theory, 2:443-467, 1994.

[8] C. Blackorby and D. Donaldson, Measures of relative equality and their meaning in terms of social welfare, Journal of Economic Theory, 18:59-80, 1978.

[9] C. Blackorby and D. Donaldson, A theoretical treatment of indices of absolute inequality, International Economic Review, 21 (1):107-136, 1980.

[10] C. Blackorby and D. Donaldson, Ethical indices for measurement of poverty, Econometrica, 48 (4):1053-1060, 1980.

[11] C. Blackorby and D. Donaldson, Ethical social index numbers and the measurement of effective tax / benefit progressivity, The Canadian Journal of Economics, 17 (4):683-694, 1984.

[12] C. Blackorby, D. Donaldson and M. Auersperg, A new procedure for the measurement of inequality within and among population subgroups, The Canadian Journal of Economics, 14 (4):665-685, 1981. 
[13] S. Bortot and R. A. Marques Pereira, The binomial Gini inequality indices and the binomial decomposition of welfare functions, Fuzzy Sets and Systems, 255:92-114, 2014.

[14] W. Bossert, An axiomatization of the singleseries Ginis, Journal of Economic Theory, 50 (1):82-92, 1990.

[15] T. Calvo, A. Kolesárova, M. Komorníková and R. Mesiar, Aggregation operators: Properties, classes and construction methods. In: $\mathrm{T}$. Calvo, G. Mayor and R. Mesiar, editors, $A g$ gregation Operators: New Trends and Applications, Physica-Verlag, Heidelberg, 2002, pp. 3-104.

[16] S. R. Chakravarty, Inequality, Polarization and Poverty: Advances in Distributional Analysis, Springer, Heidelberg, 2009.

[17] O. Chisini, Sul concetto di media, Periodico di Matematiche, 4:106-116, 1929.

[18] D. Donaldson and J. A. Weymark, A singleparameter generalization of the Gini indices of inequality, Journal of Economic Theory, 22 (2):67-86, 1980.

[19] D. Donaldson and J. A. Weymark, Ethically flexible Gini indices for income distributions in the continuum, Journal of Economic Theory, 29 (2):353-358, 1983.

[20] U. Ebert, Size and distribution of incomes as determinants of social welfare, Journal of Economic Theory, 41 (1):23-33, 1987.

[21] U. Ebert, Measurement of inequality: An attempt at unification and generalization, Social Choice Welfare, 5 (2):147-169, 1988.

[22] J. Fodor and M. Roubens, Fuzzy Preference Modelling and Multicriteria Decision Support, Kluwer Academic Publishers, Dordrecht, 1994.

[23] J. L. García-Lapresta, C. Lasso de la Vega and R. A. Marques Pereira, A class of exponential poverty measures. Proceedings of the $12^{\text {th }}$ Meeting of the Society for Social Choice and Welfare, June 18-21, Boston College, Chestnut Hill, MA, 2014.

[24] J. L. García-Lapresta, C. Lasso de la Vega, R. A. Marques Pereira and A. M. Urrutia, A class of poverty measures induced by the dual decomposition of aggregation functions, International Journal of Uncertainty, Fuzziness and Knowledge Based Systems, 18 (4):493-511, 2010.

[25] M. Grabisch, J. L. Marichal, R. Mesiar and E. Pap, Aggregation Functions, Encyclopedia of Mathematics and its Applications, Vol. 127, Cambridge University Press, 2009.

[26] A. W. Marshall and I. Olkin, Inequalities: Theory of Majorization and Its Applications, Mathematics in Science and Engineering, Academic Press, New York, 1979.

[27] N. Kakwani, On a class of poverty measures, Econometrica, 48 (2):437-446, 1980.

[28] S. C. Kolm, The optimal production of social justice. In: J. Margolis and H. Guitton, editors, Public Economics, Macmillan, London, 1969.

[29] S. C. Kolm, Unequal inequalities I, Journal of Economic Theory, 12:416-442, 1976.

[30] S. C. Kolm, Unequal inequalities II, Journal of Economic Theory, 13:82-111, 1976.

[31] F. Mehran, Linear measures of income inequality, Econometrica, 44 (4):805-809, 1976.

[32] J. Quiggin, editor, Generalized expected utility theory: the rank-dependent model, Kluwer Academic Publisher, Dordrecht, 1993.

[33] A. Sen, On Economic Inequality, Clarendon Press, Oxford, 1973.

[34] A. Sen, Poverty: an ordinal approach to measurement, Econometrica 44 (2):219-231, 1976.

[35] A. Sen, Ethical measurement of inequality: some difficulties. In: W. Krelle and A. F. Shorrocks, editors, Personal Income Distribution, North-Holland, Amsterdam, 1978.

[36] H. J. Skala, Concerning ordered weighted averaging aggregation operators, Statistical Papers, 32:35-44, 1991.

[37] J. A. Weymark, Generalized Gini inequality indices, Mathematical Social Sciences, 1 (4):409430, 1981.

[38] M. Yaari, The dual theory of choice under risk, Econometrica, 55 (1):95-115, 1987.

[39] M. Yaari, A controversial proposal concerning inequality measurement, Journal of Economic Theory, 44 (2):381-397, 1988.

[40] R. R. Yager, On ordered weighted averaging aggregation operators in multicriteria decision making, IEEE Trans. on Systems, Man and Cybernetics 18 (1):183-190, 1988.

[41] R. R. Yager and J. Kacprzyk, editors, The Ordered Weighted Averaging Operators, Theory and Applications, Kluwer Academic Publisher, Dordrecht, 1997.

[42] R. R. Yager, J. Kacprzyk and G. Beliakov, editors, Recent Developments in the Ordered Weighted Averaging Operators: Theory and Practice, Studies in Fuzziness and Soft Computing, Vol. 265, Springer, Heidelberg, 2011.

[43] S. Yitzhaki, On an extension of the Gini inequality index, International Economic Review, 24 (3):617-628, 1983. 